\title{
Quantifying electric field gradient fluctuations over polymers using ultrasensitive cantilevers
}

\author{
Showkat M. Yazdanian ${ }^{1}$, Nikolas Hoepker ${ }^{2}$, Seppe Kuehn ${ }^{1,}{ }^{*}$, Roger F. Loring ${ }^{1}$, and John A. \\ Marohn ${ }^{1, \dagger}$ \\ ${ }^{1}$ Department of Chemistry and Chemical Biology, Cornell University, Ithaca, New York 14853-1301 \\ ${ }^{2}$ Laboratory of Atomic and Solid State Physics, Cornell University, Ithaca, New York 14853
}

\section{Abstract}

An ultrasensitive cantilever, oscillating parallel to a surface in vacuum, is used to probe weak thermal electric field gradient fluctuations over thin polymer films. We measure the power spectrum of cantilever frequency fluctuations as a function of cantilever height and voltage over polymers of various compositions and thicknesses. The data are well described by a linear-response theory that calculates stochastic electric fields arising from thermally-driven dielectric fluctuations.

Development of a wide range of electrical, optical, magnetic, and biological sensors and devices requires new approaches to mapping properties of films. A large collection of scanned probe microscopies now exists for generating nanometer-resolution surface maps of chemical forces, ${ }^{1-3}$ magnetization, ${ }^{4,5}$ electrostatic potential, ${ }^{6,7}$ and capacitance. ${ }^{6,8}$ A scanned probe approach to collecting dielectric spectra has recently been demonstrated. ${ }^{9,10}$ Developments in the fabrication of attonewton-sensitivity cantilevers ${ }^{11,12}$ have created new possibilities for ultrasensitive detection and nanometer-resolution imaging of magnetic resonance, ${ }^{13,14}$ dopant density in semiconductors, ${ }^{15}$ electric field fluctuations over metals ${ }^{16}$ and in dielectric films, 17-19 and — as we will show here in experiments on polymers - electric field gradient fluctuations.

Non-contact imaging of dissipation, and hence local fluctuations, by scanned probe microscopy was first accomplished by Denk and Pohl, who detected electrical dissipation in GaAs as a sample-induced change in the mechanical quality factor of a charged atomic force microscope cantilever. ${ }^{20}$ Scanned probe studies of electrical dissipation due to eddy current damping in metals, ${ }^{21}$ bias-induced free carriers ${ }^{22,23}$ and dopant-induced free-carriers ${ }^{15}$ in semiconductors have since been reported, as has imaging of magnetic dissipation due to domain wall motion in ferromagnets. ${ }^{24,25}$

Stipe et al., in their study of the noncontact friction experienced by an ultrasensitive cantilever over a gold surface in high vacuum ${ }^{16}$ applied a fluctuation-dissipation relation connecting the noncontact friction coefficient $\Gamma$ to the fluctuating electric field $\delta E_{x}(t)$ that couples to the tip charge $q_{c}$ :

†Electronic address: jam99@cornell.edu.

*Current address: Laboratory for Living Matter, Center for Studies in Physics and Biology, The Rockefeller University, New York, New York 10065

Supporting Information Available: Details of the protocol used to determine cantilever spring constant; dielectric spectroscopy data; and data ruling out alternative frequency fluctuation mechanisms. ${ }^{19}$ This material is available free of charge via the Internet at http://pubs.acs.org. 


$$
\Gamma=\frac{q_{c}^{2}}{k_{B} T} \int_{0}^{\infty} d t \cos \left(2 \pi f_{c} t\right) C_{\delta E_{x}}(t)
$$

with $k_{B}$ Boltzmann's constant, $T$ the temperature, $f_{c}$ the cantilever frequency, $C_{\delta E_{x}}(t) \equiv$ $\left\langle\delta E_{x}(t) \delta E_{x}(0)\right\rangle$ the equilibrium correlation function of the fluctuating electric field at the cantilever tip generated by the sample, and $\delta E_{x}$ the component of the electric field parallel to the interface. These ultrasensitive measurements of noncontact friction have motivated subsequent theoretical ${ }^{26-30}$ and experimental work ${ }^{17-19,31,32}$ devoted to understanding the possible sources of electric field fluctuations over a metal sample.

Kuehn et al. used ultrasensitive cantilevers ${ }^{11,12}$ in a non-contact friction measurement to quantify fluctuating electric fields over polymer samples. ${ }^{17}$ These measurements were analyzed by calculating $\Gamma$ from eq 1 , with $C_{\delta E_{x}}(t)$ determined from a dielectric continuum model. ${ }^{18,19}$ In this picture, the cantilever friction arises from thermal dielectric fluctuations in the polymer film, and $\Gamma$ is determined by the polymer's complex-valued relative dielectric function at the cantilever frequency. Using measured values of the dielectric function, and an estimate of the tip-sample capacitance to relate the tip-sample voltage to $q_{c}$, Kuehn et al. showed that eq 1 agreed well with the measured friction for three polymer species over a range of sample thicknesses and probe heights.

Israeloff and coworkers were the first to access the dielectric spectrum of a polymer by means of scanned-probe microscopy. They observed cantilever frequency fluctuations (jitter) above the surfaces of thin polymer films in vacuum. ${ }^{33-} 35$ They interpreted these cantilever frequency fluctuations as generated by a fluctuating electrostatic potential arising from thermal dynamics in the film. In their analysis, the power spectrum of cantilever frequency fluctuations was related to the absorptive part of the dielectric function of the film, $33^{-} 35$ but the dependence of frequency noise on probe height and film thickness was not explicitly given.

An important difference between the measurements of Israeloff and coworkers ${ }^{33-35}$ and those presented here is the experimental geometry. In the measurements of Refs. ${ }^{33-35}$, the cantilever tip moves perpendicular to the vacuum-polymer interface, as in most scanned-probe experiments, while in the measurements reported here, the cantilever tip moves parallel to the vacuum-polymer interface, as sketched in Fig. 1(a). In the case of perpendicular tip motion, the tip-sample capacitance and hence the tip charge $q_{c}$ can vary significantly over the period of the probe's motion, while for parallel tip motion, $q_{c}$ may be assumed to be constant over the period of the probe's motion. Yazdanian et al. recently reported an approach to calculating both cantilever frequency noise and noncontact friction in terms of the dielectric function, film thickness, and probe height. ${ }^{19}$ Calculations in Ref. ${ }^{19}$ were performed for the case of parallel tip motion, and the frequency jitter was found to be driven by fluctuations in the electric field gradient at the tip.

Generalizing this analysis to the case of perpendicular tip motion, we have found that the frequency jitter includes contributions from three mechanisms: (1) voltage fluctuations that couple to the second spatial derivative of the capacitance, as invoked by Israeloff and coworkers, $33^{-} 35$ (2) electric field fluctuations that couple to the gradient of the capacitance, and (3) fluctuations in the electric field gradient that couple to the tip charge as for the parallel geometry. If the spatial derivatives of the capacitance are negligible as for the case of the parallel geometry, only mechanism (3) remains. Thus frequency jitter with the perpendicular geometry of Refs. $33^{-} 35$ and frequency jitter with the parallel geometry as reported here are actually different experimental observables with different information content. 
For the configuration of Figure 1(a), we have shown ${ }^{19}$ that the power spectrum of cantilever frequency fluctuations $P_{\delta f_{c}}(f)$ can be written as

$$
\begin{array}{r}
P_{\delta f_{c}}(f) \\
=\left(\frac{q_{c}^{2} f_{c}^{2}}{k_{c}^{2}}\right) \int_{0}^{\infty} d t \cos (2 \pi f t) C_{\delta E_{x x}}(t),
\end{array}
$$

where we define the power spectrum as

$$
\begin{array}{r}
P_{\delta f_{c}}(f) \\
=4 \int_{0}^{\infty} d t \cos (2 \pi f t)\left\langle\delta f_{c}(t) \delta f_{c}(0)\right\rangle .
\end{array}
$$

Here $k_{c}$ is the cantilever spring constant and $C_{\delta E_{x x}}(t) \equiv\left\langle\delta E_{x x}(t) \delta E_{x x}(0)\right\rangle$ is the equilibrium autocorrelation function of fluctuations in $E_{x x} \equiv \partial E_{x} / \partial x$, the electric field gradient at the probe tip. In Ref. ${ }^{19}$ we evaluated $C_{\delta E_{x x}}(t)$ from a dielectric continuum model for the polymer film and found

$$
\begin{array}{r}
P_{\delta f_{c}}(f) \\
=\left(\frac{3 k_{B} T f_{c}^{2} q_{c}^{2}}{64 \pi^{2} \varepsilon_{0} k_{c}^{2} d^{5}}\right)\left(\frac{\operatorname{Im} \zeta(2 \pi f) J_{4}(2 \pi f)}{f}\right), \\
J_{4}(2 \pi f)=\int_{0}^{\infty} d p p^{4}\left(\frac{e^{-2 p}\left(1-e^{-4 p h / d}\right)}{\left|1+\zeta(2 \pi f) e^{-2 p h / d}\right|^{2}}\right), \\
\zeta(2 \pi f) \equiv \frac{\varepsilon(2 \pi f)-1}{\varepsilon(2 \pi f)+1} .
\end{array}
$$

The jitter power spectrum is related here to the tip-sample separation $d$, the sample thickness $h$, the cantilever force constant $k_{c}$ and frequency $f_{c}$ in the absence of the sample, the tip charge $q_{c}$, and the complex-valued relative dielectric function of the sample $\varepsilon(2 \pi f)$. For known tip charge, $q_{c}$, this expression contains no free parameters. The purpose of this paper is to make a rigorous comparison of eqs 4-6 to new measurements of the power spectrum of cantilever frequency fluctuations over polymer films of various composition as a function of height and film thickness.

Measurements were carried out with custom-fabricated silicon microcantilevers ${ }^{11,12,17}$ whose sensitivity is two orders of magnitude higher than commercial cantilevers. Figure 1(b) shows a scanning electron micrograph of a representative cantilever, made from single-crystal silicon. The cantilevers are $L=275 \mu \mathrm{m}$ long, $7 \mu \mathrm{m}$ wide, and $340 \mathrm{~nm}$ thick, with spring constants between $7.6 \times 10^{-4}$ and $1.7 \times 10^{-3} \mathrm{~N} / \mathrm{m}$, resonance frequencies between 5 and $6 \mathrm{kHz}$, and mechanical quality factors as high as 15000 in high vacuum $\left(P=10^{-6} \mathrm{mbar}\right)$. By offsetting the optical mask during lithography, ${ }^{11,17}$ a tip width as small as $\sim 100 \mathrm{~nm}$ could be achieved. The leading edge of the cantilever was thinned to $150 \mathrm{~nm}$ and coated with $15 \mathrm{~nm}$ of platinum using 
a shadow mask. ${ }^{11,17}$ The resulting metallized cantilever tip is reasonably well approximated as a sphere. A tip before metallization can be seen in the inset of Fig. 1(b).

The spring constant $k_{c}$ of each cantilever was measured by analyzing thermomechanical position fluctuations using the equipartition theorem. ${ }^{36}$ The mean-square amplitude of cantilever thermal vibrations, $\left\langle x_{\mathrm{th}}^{2}\right\rangle$, was estimated from the area under an averaged positionfluctuation power spectrum, calculated from 25 transients lasting $25 \mathrm{~s}$ each. The spring constant was then computed as $k_{c}=k_{B} T /\left\langle x_{\mathrm{th}}^{2}\right\rangle$.

Films were prepared of three polymers: poly(methyl methacrylate), poly(vinyl acetate), and polystyrene (Table I). The polymers were spin-cast from toluene onto epitaxial Au(111)-onmica substrates (Agilent; Part No. N9805B-FG) and their thickness measured by profilometry. The samples were then transferred to a high vacuum oven $\left(P=10^{-6} \mathrm{mbar}\right)$ and annealed at $T_{g}+10^{\circ} \mathrm{C}$ for 12 hours to remove solvent and water. For $T_{g}$, we used glass transition temperatures published for polymers with molecular weights similar to ours. ${ }^{37-39}$ Dielectric spectra, $\varepsilon(2 \pi f)$, for PMMA and PVAc were measured in $440 \mathrm{~nm}$ thick polymer films using an impedance analyzer (Hewlett Packard; Model No. 4192 A LF) ${ }^{40}$ The spectrum of polystyrene was kindly provided by Professor Ranko Richert.41

To study each film, a cantilever was mounted to a custom-built scanning probe microscope. 19,40 Cantilever displacement was detected with an optical fiber interferometer operating at $1310 \mathrm{~nm}$. The microscope had two levels of vibration isolation - custom-made stainless steel bellows and a commercial vibration isolation stage (Minus- $k$ 250BM-3). A commercial nanopositioner (Attocube ANPz 51) was used to bring the cantilever into contact with the surface and to scan the tip-sample separation. The film surface $(d=0)$ was located by measuring cantilever quality factor $Q$, via a ringdown measurement, at various heights $d$; the surface was defined within $\pm 2 \mathrm{~nm}$ as the height at which $Q$ extrapolated to zero. ${ }^{16,17,40}$ This noncontact protocol for locating the surface was validated by bringing the cantilever towards the surface and watching with the interferometer for a characteristic dc displacement that occurs at $d=0$ when the cantilever buckles upon touching the surface.

A cantilever was then brought to a calibrated distance $d$ over a film and its tip charged by applying a voltage $V_{\text {ts }}$ between the cantilever and the gold of the substrate. To measure temporal fluctuations in the cantilever resonance frequency over a film, the cantilever was driven into self oscillation using a custom-built analog positive feedback loop..$^{42}$ The resulting oscillation $x(t)$ was digitized and passed through a $10^{\text {th }}$ order Butterworth bandpass filter (center frequency $f_{c}$, bandwidth $f_{\text {filt }}=2 \times 2100 \mathrm{~Hz}$ ). The instantaneous cantilever frequency $f_{c}(t)$ was determined from the filtered signal by means of a software frequency demodulator, as described in detail in the Supplement of Ref. ${ }^{19}$. Averaged power spectra $P_{\delta f_{c}}$ of cantilever frequency fluctuations $\delta f_{c}(t)=f_{c}(t)-\left\langle f_{c}\right\rangle$ were determined using Eq. 3 ; each spectrum was computed from, typically, $N_{\text {avg }}=50$ frequency transients lasting $T_{\text {acq }}=25 \mathrm{~s}$ each. Bandpass filtering $x(t)$ has the effect of lowpass filtering $P_{\delta f_{c}}$. Using a high order filter is important to avoid contamination of low $f$ Fourier components in $P_{\delta f}$ by aliasing of instrument noise present on either side of $f_{c}$ in $x(t)$. The non-contact friction coefficient was measured ${ }^{17}$ by recording the cantilever ringdown time $\tau$ and calculating $\Gamma=k_{c} / 2 \pi^{2} f_{c}^{2} \tau$.

The data of Figure 2(a-b) confirm ${ }^{33-35}$ that a charged cantilever does indeed exhibit increased frequency fluctuations near the surface of a polymer film. The sample is a $200 \mathrm{~nm}$ thick film of PMMA. As the tip-sample separation is decreased from $d=2000 \mathrm{~nm}$ to $d=25 \mathrm{~nm}$, the rootmean-square fluctuation in cantilever frequency increases over fourfold. With an ultrasensitive cantilever, this increase in frequency noise due to fluctuations in the film can be easily distinguished even with only $1 \mathrm{~s}$ of acquisition. 
To better distinguish polymer-induced cantilever frequency noise from contributions originating in cantilever thermomechanical position fluctuations and detector noise, it is useful to examine the power spectrum of cantilever frequency fluctuations. In Fig. 2(c) we show the power spectrum of cantilever frequency fluctuations, $P \delta f_{c}(f)$ vs. $f$, at a height $d=2000 \mathrm{~nm}$ over a $200 \mathrm{~nm}$ thick film of PMMA. The horizontal dot-dashed line is the contribution to frequency noise expected from thermomechanical position fluctuations ${ }^{19,42,43}$ :

$$
P_{\delta f_{c}}^{\text {therm }}=\frac{k_{B} T f_{c}}{2 \pi x_{\mathrm{rms}}^{2} k_{c} Q}
$$

where $x_{\mathrm{rms}}$ is the root-mean-square amplitude of the driven cantilever. The dashed line in the figure is the detector's contribution to frequency noise ${ }^{19,42,43}$ :

$$
P_{\delta f_{c}}^{\mathrm{det}}=\frac{P_{\delta x}^{\mathrm{det}}}{x_{\mathrm{rms}}^{2}} f^{2}
$$

where $P_{\delta x}^{\mathrm{det}}$ is detector noise written in terms of an equivalent fluctuation of the cantilever displacement. In the absence of a sample (or at large tip-sample separations), we find cantilever frequency fluctuations to be dominated by thermomechanical position fluctuations and detector noise, as seen in Fig. 2(c). Equations 7 and 8 together account, quantitatively, for the entire power spectrum of cantilever frequency fluctuations apparent in Fig. 2(c) at large tip-sample separation.

At small tip-sample separations, in contrast, the power spectrum of cantilever frequency fluctuations is much larger than expected from thermomechanical and detector noise alone. In Fig. 2(d) we show the power spectrum of cantilever frequency fluctuations at a height $d=$ $25 \mathrm{~nm}$. Near $f=0$, the frequency fluctuations have a power spectrum over $10^{2}$ times larger than the thermomechanical and detector contributions. We attribute this excess frequency noise to tip-sample interactions. At $d=25 \mathrm{~nm}$, with our ultrasensitive cantilevers, we can resolve Fourier components in sample-induced frequency noise up to a frequency of approximately $f$ $=500 \mathrm{~Hz}$, a factor of twenty higher than was demonstrated in Ref. ${ }^{35}$.

Having established that polymer-induced frequency fluctuations are large and detectable with excellent signal-to-noise using an ultrasensitive cantilever oscillating parallel to the sample surface, we are now ready to explore the dependence of frequency fluctuations on tip charge, tip-sample separation, and film composition and thickness. We will summarize these studies by plotting the frequency integral of the power spectrum of frequency fluctuations,

$$
\int_{f_{\mathrm{L}}}^{f_{\mathrm{U}}} d f P_{\delta f_{c}}(f)
$$

We refer to this quantity as "jitter". In the hypothetical limit that $f_{\mathrm{L}} \rightarrow 0$ and $f_{\mathrm{U}} \rightarrow \infty$, this quantity equals $\left\langle\left(\delta f_{c}\right)^{2}\right\rangle$. We set the lower cutoff frequency to $f_{\mathrm{L}}=0 \mathrm{~Hz}$ except when comparing theory to experiment, in which case we set $f_{\mathrm{L}}=5 \mathrm{~Hz}$ to reject low-frequency noise in the measured dielectric function. The upper cutoff frequency, $f_{\mathrm{U}}=100 \mathrm{~Hz}$, is necessary to reject high-frequency instrument noise present in the measurements (Fig. 2(c-d)).

In Fig. 2(e) we plot jitter at height $d=2000 \mathrm{~nm}$ over a PMMA film as a function of cantilever amplitude. The solid line on the log-log plot has slope -2 , confirming that jitter $\propto 1 / x_{\mathrm{rms}}^{2}$, as 
expected from eq 7. The jitter vs. cantilever amplitude at small tip-sample separation, $d=25 \mathrm{~nm}$, can be seen in Fig. 2(f). At small cantilever amplitude, the jitter is approximately constant (solid line), in agreement with the continuum model of dielectric fluctuations used to derive eqs 4-6. At large $x_{\text {rms }}$, however, the jitter in Fig. 2(f) decreases substantially. By introducing a wavevector-dependent dielectric constant, $\varepsilon \circ(\boldsymbol{k}, \omega)$, into the theory of Ref. ${ }^{19}$, we believe it should be possible to use data like that in Fig. 2(f) to obtain information about spatial-temporal correlations in the sample's dielectric function - an exciting possibility.

In Fig. 3 we plot frequency jitter vs. tip-sample voltage for a cantilever at various heights over a PMMA film. The applied voltage puts a charge on the tip $q_{c}=C\left(V_{\mathrm{ts}}-\varphi\right)$, where $C$ is the tipsample capacitance and $\varphi$ is the difference in contact potential between tip and sample. From eqs 2 and 9 we expect jitter $\propto C^{2}\left(V_{\mathrm{ts}}-\varphi\right)^{2}$. We can see in Fig. 3 that the jitter is indeed a quadratic function of the applied tip voltage. By fitting the data to a parabola we can extract the contact potential $\varphi$. We note that measured $\varphi$ is not quite independent of tip-sample separation as one might expect; we will return to this observation below in discussing Fig. 6 . That jitter depends quadratically on voltage confirms a key assumption of our theory ${ }^{19}$ — that cantilever frequency fluctuations can be calculated using linear-response theory.

We now compare the measured $P_{\delta f_{c}}$ to eq 4 . In order to calculate $q_{c}$, we approximate the charged tip of the cantilever as a sphere of radius $R$ located a distance $d$ above a dielectric slab of thickness $h$ backed by a conductor. ${ }^{19,44,45}$ We use eqs 6.5 and 6.6 in Ref. ${ }^{19}$ to calculate the tip-sample capacitance $C$ and then calculate the tip charge $q_{c}$ from $C$, the measured contact potential $\varphi$, and the applied tip-sample voltage $V_{\mathrm{ts}}$. We take the tip radius to be $R=70 \mathrm{~nm}$, based on the scanning electron micrograph in Fig. 1(b).

In Fig. 4 we compare the measured and calculated $P_{\delta f_{C}}$ at a height $d=100 \mathrm{~nm}$ above a $200 \mathrm{~nm}$ thick PMMA film. The measured and calculated curves agree within better than a factor of two over more than two decades of frequency. A $P_{\delta f_{c}} \propto 1 / f$ line is plotted for comparison. We see that, in this experiment, the cantilever frequency-fluctuation power spectrum is well approximated as a $1 / f$ spectrum for frequencies in the 5 to $500 \mathrm{~Hz}$ range, with small deviations from $1 / f$ behavior apparent only below $f \approx 5 \mathrm{~Hz}$.

This finding is in qualitative agreement with the $P_{\delta f_{c}} \propto \varepsilon^{\prime \prime}(f) / f$ behavior predicted by Walther et al. ${ }^{33}$ given that the measured $\varepsilon^{\prime \prime}$ for our PMMA sample is approximately constant from $f=$ $5 \mathrm{~Hz}$ to $500 \mathrm{~Hz}$. Equations 4-6 also predict $P_{\delta f_{c}} \varepsilon^{\prime \prime}(f) / f$ for the limiting case $\varepsilon^{\prime \prime}(f) \ll 1$, valid for our measurements. Equations 4-6 predict quantitatively the absolute size of the observed frequency fluctuations

In Fig. 5 we use the measured contact potential, estimated capacitance, measured dielectric spectrum, and eqs 4 and 9 to predict jitter in a bandwidth from 5 to $100 \mathrm{~Hz}$ as a function of tipsample separation $d$. In computing tip charge, we found it necessary to account for the height dependence of the contact potential $\varphi$ shown in Fig. 3, as follows. In each sample $\varphi$ was measured at 4 to 10 selected heights. Over the six samples studied at heights from $d=10 \mathrm{~nm}$ to over $200 \mathrm{~nm}$, the observed $\varphi$ ranged from $0.29 \mathrm{~V}$ to $0.95 \mathrm{~V}$. In any one sample, a variation of $\leq 0.2 \mathrm{~V}$ was seen. In each sample, a high, low, and average contact potential ( $\varphi_{\text {high }}, \varphi_{\text {low }}$, and $\varphi_{\text {avg }}$ ) were identified. The applied voltage was set to $V_{\mathrm{ts}} \approx 0.5 \mathrm{~V}+\varphi_{\text {avg. }}$. To account for the observed variation in contact potential, for each sample two theory curves are calculated: one with $\varphi=\varphi_{\text {low }}$ and one with $\varphi=\varphi_{\text {high. }}$. In Fig. 5 the region between these two curves is colored yellow.

The results for six samples are shown in Fig. 5: films of PS (upper row), PVAc (middle row), and PMMA (lower row) of thickness $h=40 \mathrm{~nm}$ (left column) and $450 \mathrm{~nm}$ (right column). Theory correctly predicts the magnitude of the observed jitter and its dependence, qualitatively, on 
distance. Comparing films of the same composition, theory also predicts correctly whether the thick film or the thin film will have the larger jitter at close separation. This prediction cannot be made without a detailed calculation: the electric field gradient fluctuations are largest over the thick film, but at fixed tip-sample voltage the tip charge is less over the thick film and so the fluctuations couple less well to the cantilever; differences in $k_{c}$ must also be accounted for.

The agreement between theory and experiment is poorest in polystyrene, where theory underestimates the jitter observed at close separations by a factor of three in the thick film and by a factor of ten in the thin film. It is not surprising that of the three samples, polystyrene would show the worst agreement. The $\varepsilon^{\prime \prime}$ in polystyrene is very small and notoriously difficult to measure, and is likely therefore more sensitive to sample preparation than either PMMA and PVAc. Although we were able to obtain $\varepsilon^{\prime}$ and $\varepsilon^{\prime \prime}$ for polystyrene of molecular weight comparable to ours, ${ }^{41}$ the sample was not identical to that used in our cantilever measurements. Neither $\varepsilon^{\prime}$ nor $\varepsilon^{\prime \prime}$ for PS depend significantly on frequency over the relevant range. Treating these constants as adjustable parameters does not yield a dielectric function that reproduces the PS data for both thin and thick films with the same level of agreement shown for PVAc and PMMA. This suggests that dielectric fluctuations in the thin PS film are not well represented by bulk dielectric relaxation processes alone.

For the four PMMA and PVAc films, where $\varepsilon^{\prime}$ and $\varepsilon^{\prime \prime}$ could be measured in identically-prepared samples, the measured and predicted jitter are in quantitative agreement. The magnitude of the jitter and its dependence on tip-sample separation is correctly predicted over two decades of jitter in both of these samples. The agreement between theory and measurement in Fig. 5 confirms ${ }^{33-35}$ that the jitter measurement probes low frequency polymer motions. In PMMA, for example, these low frequency motions include hindered rotations of polar side groups. ${ }^{46}$

The ability to detect minute electric field gradient fluctuations is greatly enhanced by using an ultrasensitive cantilever. To demonstrate this, let us define a spectral density of electric field gradient fluctuations $P_{\delta E_{x x}}$ by analogy with eq 3 and rewrite $P_{\delta f_{c}}$ (eq 2) in terms of $P_{\delta E_{x x}}$. To determine the thermally-limited minimum detectable electric field gradient, we set $P_{\delta f_{C}}$ equal to $P_{\delta f_{c}}^{\text {therm }}$ (eq 7),

$$
P_{\delta E_{x x}}^{\mathrm{therm}}=\frac{4 k_{B} T \Gamma}{q_{c}^{2} x_{\mathrm{rms}}^{2}}
$$

where we have written the result in terms of the dissipation constant using $\Gamma=k_{c}=2 \pi f_{c} Q$. For a commercial electric force microscope cantilever, $\Gamma=1.5 \times 10^{-9} \mathrm{Ns} / \mathrm{m}\left(f_{c}=75 \mathrm{kHz}, k_{c}=3.5\right.$ $\mathrm{N} / \mathrm{m}$, and $Q=5 \times 10^{3}$ ). For an ultrasensitive cantilever, the friction coefficient can be as good as ${ }^{17} \Gamma=5 \times 10^{-13} \mathrm{Ns} / \mathrm{m}\left(f_{c}=7.4 \mathrm{kHz}, k_{c}=0.7 \mathrm{mN} / \mathrm{m}\right.$, and $\left.Q=3.1 \times 10^{4}\right)$. We conclude that the ultrasensitive cantilever can resolve a 3000-fold smaller fluctuation $P_{\delta E_{x x}}$ than can a commercial cantilever operated in vacuum with the same tip charge and amplitude.

Above we inferred the contact potential $\varphi$ from a plot of jitter vs. voltage acquired at selected tip-sample separations. We explored a much quicker, and in principle equivalent, method for measuring $\varphi$ : recording frequency as a function of applied tip voltage. The voltage dependence of the non-contact friction coefficient was measured simultaneously as a control.

Figure 6(a) shows a plot of jitter, noncontact friction coefficient $\Gamma$, and frequency $f_{c}$ over a PMMA film as a function of tip-sample voltage $V_{\mathrm{ts}}$. The contact potential obtained by fitting the jitter vs. voltage data to a parabola is $\varphi_{\text {jitter }}=-0.14 \pm 0.02 \mathrm{~V}$. Fitting the $\Gamma$ vs. $V_{\text {ts }}$ curve to a parabola gives a statistically equivalent result: $\varphi_{\Gamma}=-0.13 \pm 0.04 \mathrm{~V}$. While the cantilever 
frequency is also observed to be a parabolic function of voltage, the contact potential inferred from the $f_{c}$ vs. $V_{\mathrm{ts}}$ curve is quite different: $\varphi_{f_{c}}=-1.02 \pm 0.03 \mathrm{~V}$, twenty four standard deviations away from $\varphi_{\text {jitter }}$ and $\varphi_{\Gamma}$.

Parabolas of jitter, $\Gamma$, and frequency $f_{c}$ were used to infer $\varphi_{\mathrm{jitter}}, \varphi_{\Gamma}$, and $\varphi_{f_{c}}$ as a function of tipsample separation for $d=25$ to $500 \mathrm{~nm}$ over the PMMA sample. The results are summarized in Fig. 6(b). The contact potentials inferred from jitter and noncontact friction agreed within $0.1 \mathrm{~V}$ over the range of $d$ and were essentially independent of tip-sample separation. While $\varphi_{f_{c}}$ agrees with $\varphi_{\mathrm{jitter}}$ and $\varphi_{\Gamma}$ at very small tip-sample separations, it differs from $\varphi_{\mathrm{jitter}}$ and $\varphi_{\Gamma}$ by as much as $1.5 \mathrm{~V}$ at a few hundred nanometer separation.

A similarly large height-dependent shift in contact potential has been seen by Rast $e t a l .{ }^{31}$ in measurements of the frequency of an ultrasensitive cantilever over a gold film in ultrahigh vacuum. This shift was ascribed ${ }^{31}$ to either patch charges ${ }^{47}$ on the gold or to surface contaminants. Neither hypothesis explains the discrepancy between $\varphi_{f_{c}}$ and $\varphi_{\mathrm{jitter}}$ or $\varphi_{\Gamma}$ seen in Fig. 6. Silveira et al. have suggested that in an experiment with the cantilever oscillating perpendicular to the surface at constant voltage, the force (e.g., displacement) and frequency parabolas could have different minima in the presence of a height-dependent contact potential. ${ }^{48}$ An alternative hypothesis is that the frequency measurement is more sensitive to the presence of charge on the silicon body of the cantilever, which has a different work function from the platinum tip, than are the measurements of friction and jitter.

Figures 4 and 5 are the central results of this letter. They show that our linear-response theory 19 accurately predicts the dependence of cantilever frequency noise on polymer composition, tip-sample separation, and film thickness, as well as predicting the shape of the frequency-fluctuation power spectrum. The inputs to the theory - the polymer's lowfrequency dielectric spectrum and the tip diameter, used to estimate the capacitance - were measured independently. The comparison to theory thus involves no free parameters. Taken together with the results of Refs. 17 and ${ }^{18}$, the data presented here suggest that a single unified theory describes noncontact friction (due to electric field fluctuations) and frequency jitter (due to electric field gradient fluctuations) in thin polymer films.

Cantilever dissipation probes only the Fourier component of electric field fluctuations at the cantilever frequency. ${ }^{16,17,40}$ In contrast, the entire low frequency spectrum of electric field gradient fluctuations is encoded in cantilever frequency noise. ${ }^{33-35,49}$ Our results show that ultrasensitive cantilevers are a particularly broadband detector, capable of quantifying electric field gradient fluctuations from below $10^{-2} \mathrm{~Hz}$ to nearly $10^{3} \mathrm{~Hz}$. Operating the cantilever in the parallel geometry of Fig. 1 makes it possible to straightforwardly interpret frequency noise in terms of an electric field gradient fluctuation. Our findings motivate using an ultrasensitive cantilever to test microscopic models of other fluctuations, such as charge fluctuations in heterogeneous electronic materials.

\section{Supplementary Material}

Refer to Web version on PubMed Central for supplementary material.

\section{Acknowledgments}

We thank Professor Ranko Richert for a polystyrene dielectric spectrum. SMY acknowledges the National Science Foundation through the Cornell Center for Nanoscale Systems (EEC-0117770 and EEC-0646547) and grants DMR-0134956 and DMR-0706508. NH acknowledges the U.S. Army Research Office Multi-University Research Initiative (W911NF-05-1-0403) and the Principality of Liechtenstein. SK acknowledges the National Institutes of Health (5R01GM-070012) and the Society of Analytical Chemists of Pittsburgh. RFL acknowledges the National Science Foundation (CHE-0413992 and CHE-0743299). JAM acknowledges the National Institutes of Health (5R01GM-070012), the Army Research Office Multi-University Research Initiative (W911NF-05-1-0403), and the 
National Science Foundation (DMR-0134956). This work was performed in part at the Cornell NanoScale Science and Technology Facility, a member of the National Nanotechnology Infrastructure Network, supported by the National Science Foundation (ECS-0335765).

\section{References}

1. Binnig G, Quate C, Gerber C. Phys Rev Lett 1986;56:930 -933. [PubMed: 10033323]

2. Martin Y, Williams C, Wickramasinghe H. J Appl Phys 1987;61:4723 - 4729.

3. Frisbie C, Rozsnyai L, Noy A, Wrighton M, Lieber C. Science 1994;265:2071 - 2074. [PubMed: 17811409]

4. Martin Y, Wickramasinghe H. Appl Phys Lett 1987;50:1455 -1457.

5. Rugar D, Mamin H, Guethner P, Lambert SE, Stern J, McFadyen I, Yogi T. J Appl Phys 1990;68:1169 $-1183$.

6. Martin Y, Abraham D, Wickramasinghe H. Appl Phys Lett 1988;52:1103 -1105.

7. Nonnenmacher M, O’Boyle M, Wickramasinghe H. Appl Phys Lett 1991;58:2921 - 2923.

8. Kalinin, S.; Gruverman, A. Scanning Probe Microscopy: Electrical and Electromechanical Phenomena at the Nanoscale. Springer Verlag; New York: 2005.

9. Crider PS, Majewski MR, Zhang J, Ouckris H, Israeloff NE. Appl Phys Lett 2007;91:013102.

10. Crider PS, Majewski MR, Zhang J, Ouckris H, Israeloff NE. J Chem Phys 2008;128:044908. [PubMed: 18248001]

11. Stowe TD, Yasumura K, Kenny TW, Botkin D, Wago K, Rugar D. Appl Phys Lett 1997;71:288 290.

12. Jenkins NE, DeFlores LP, Allen J, Ng TN, Garner SR, Kuehn S, Dawlaty JM, Marohn JA. J Vac Sci Technol B 2004;22:909 - 915.

13. Rugar D, Budakian R, Mamin HJ, Chui BW. Nature 2004;430:329 - 332. [PubMed: 15254532]

14. Kuehn S, Hickman SA, Marohn JA. J Chem Phys 2008;128:052208. [PubMed: 18266413]

15. Stowe TD, Kenny TW, Thomson DJ, Rugar D. Appl Phys Lett 1999;75:2785 - 2787.

16. Stipe BC, Mamin HJ, Stowe TD, Kenny TW, Rugar D. Phys Rev Lett 2001;8709:6801.

17. Kuehn S, Loring RF, Marohn JA. Phys Rev Lett 2006;96:156103. [PubMed: 16712172]

18. Kuehn S, Marohn JA, Loring RF. J Phys Chem B 2006;110:14525 - 14528. [PubMed: 16869550]

19. Yazdanian SM, Marohn JA, Loring RF. J Chem Phys 2008;128:224706. [PubMed: 18554042]

20. Denk W, Pohl DW. Appl Phys Lett 1991;59:2171 - 2173.

21. Hoffmann B, Houbertz R, Hartmann U. Appl Phys A 1998;66:S409 - S413.

22. Park JY, Ogletree DF, Thiel PA, Salmeron M. Phys Rev B 2007;76:064108.

23. Qi Y, Park JY, Hendriksen BLM, Ogletree DF, Salmeron M. Phys Rev B 2008;77:184105.

24. Grütter P, Liu Y, LeBlanc P, Durig U. Appl Phys Lett 1997;71:279 - 281.

25. Liu Y, Grütter P. J Appl Phys 1998;83:5922 - 5926.

26. Volokitin A, Persson B. Phys Rev Lett 2003;91:106101. [PubMed: 14525493]

27. Zurita-Sanchez J, Greffet J, Novotny L. Phys Rev A 2004;69:022902.

28. Chumak A, Milonni P, Berman G. Phys Rev B 2004;70:085407.

29. Volokitin A, Persson B. Phys Rev Lett 2005;94:086104. [PubMed: 15783908]

30. Volokitin A, Persson B, Ueba H. Phys Rev B 2006;73:165423.

31. Rast S, Gysin U, Ruff P, Gerber C, Meyer E, Lee D. Nanotechnology 2006;17:S189 - S194.

32. Labaziewicz J, Ge Y, Leibrandt DR, Wang SX, Shewmon R, Chuang IL. Phys Rev Lett 2008;101:180602. [PubMed: 18999810]

33. Walther LE, Russell EV, Israeloff NE, Gomariz HA. Appl Phys Lett 1998;72:3223 - 3225.

34. Russell EV, Israeloff NE, Walther LE, Gomariz HA. Phys Rev Lett 1998;81:1461 -1464.

35. Russell EV, Israeloff NE. Nature 2000;408:695 - 698. [PubMed: 11130066]

36. Hutter J, Bechhoefer J. Rev Sci Instrum 1993;64:1868 -1873.

37. Keddie J, Jones R, Cory R. Faraday Discuss 1994;98:219 -230. 
38. Fukao, K.; Uno, S.; Miyamoto, Y.; Hoshino, A.; Miyaji, H. J Non-Cryst Solids. 2002. p. 307-310.p. 517-523.

39. Wada Y, Hirose H, Asano T, Fukutomi S. J Phys Soc Japan 1959;14:1064 -1072.

40. Kuehn, S. Ph D Thesis. Cornell University; New York: 2007. Force-gradient detected nuclear magnetic resonance and the origins of noncontact friction.

41. Richert R. (personal communication).

42. Albrecht TR, Grütter P, Horne D, Rugar D. J Appl Phys 1991;69:668 - 673.

43. Obukhov Y, Fong KC, Daughton D, Hammel PC. J Appl Phys 2007;101:034315.

44. Terris BD, Stern JE, Rugar D, Mamin HJ. Phys Rev Lett 1989;63:2669 - 2672. [PubMed: 10040956]

45. Cherniavskaya O, Chen L, Weng V, Yuditsky L, Brus LE. J Phys Chem B 2002;107:1525 - 1531.

46. Bergman R, Alvarez F, Alegria A, Colmenero J. J Chem Phys 1998;109:7546 - 7555.

47. Burnham NA, Colton RJ, Pollock HM. Phys Rev Lett 1992;69:144 -147. [PubMed: 10046210]

48. Silveira, WR.; Muller, EM.; Ng, T-N.; Dunlap, DH.; Marohn, JA. Scanning Probe Microscopy:

Electrical and Electromechanical Phenomena at the Nanoscale. Kalinin, SV.; Gruverman, A., editors. Vol. II. Springer; New York: 2007. Chapter "High-sensitivity electric force microscopy of organic electronic materials and devices":788.

49. Crider PS, Israeloff NE. Nano Lett 2006;6:887 - 889. [PubMed: 16683819] 


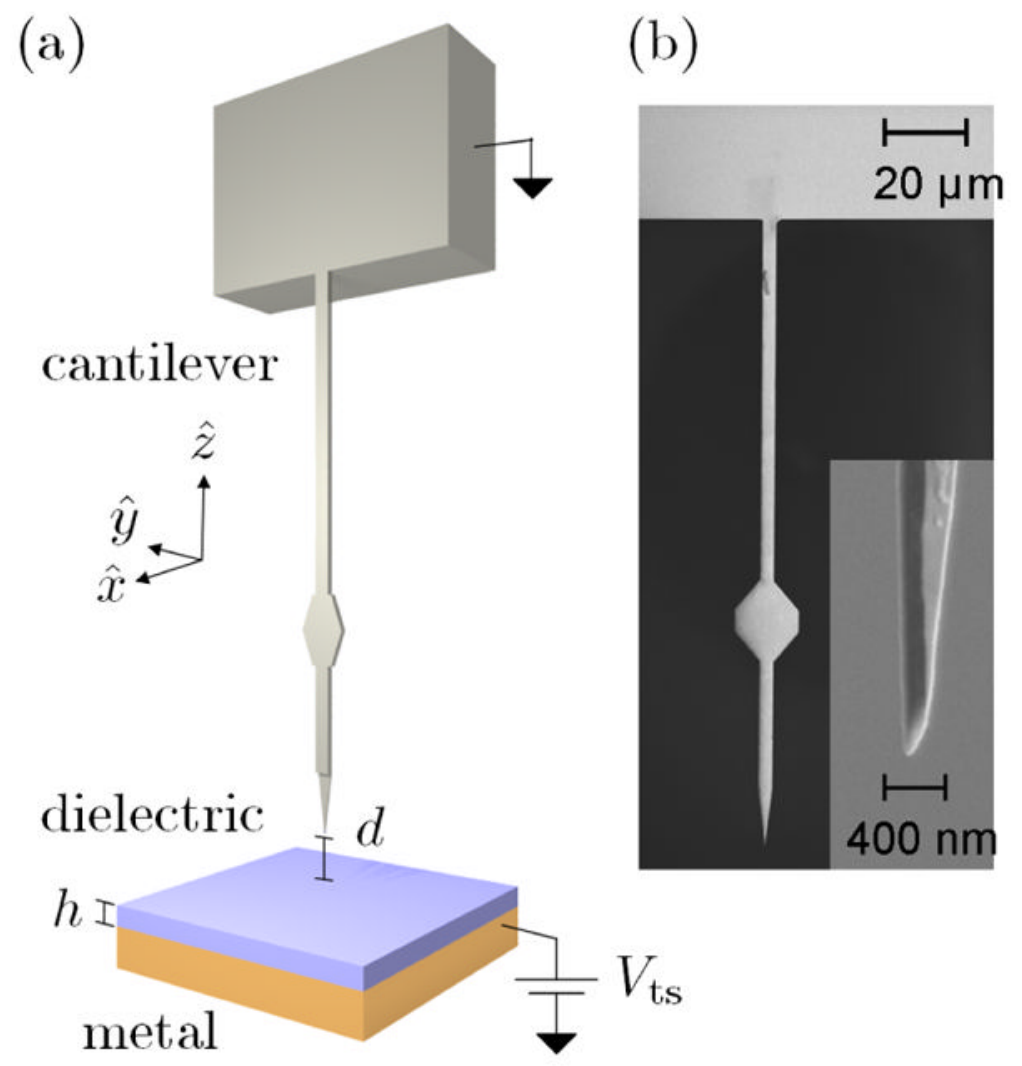

FIG. 1.

Experimental set-up. (a) A charged cantilever tip oscillates in the $x$ direction, parallel to the sample surface, at a height $z=d$ above the surface of a dielectric sample of thickness $h$. The tip is charged by applying a voltage $V_{\mathrm{ts}}$ between the tip and a metal film under the sample. (b) Scanning electron micrograph of a representative cantilever. The hexagonal pad acts as a target for an optical fiber interferometer. Inset: Close-up of the cantilever tip. Here the cantilever is shown prior to coating its end with platinum using a shadow mask. 
(a)

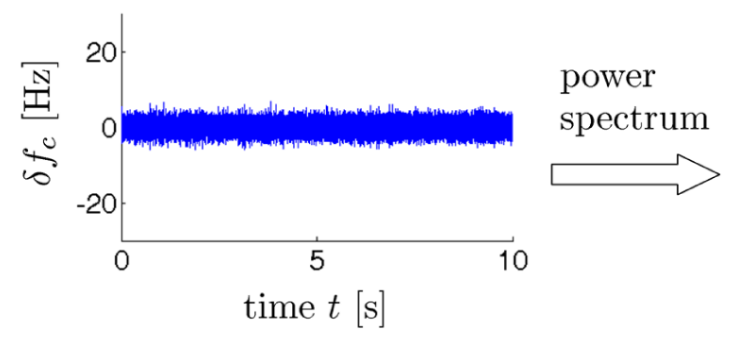

(b)
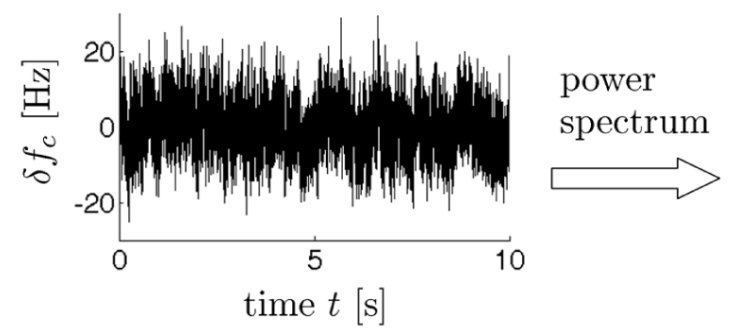

(c)

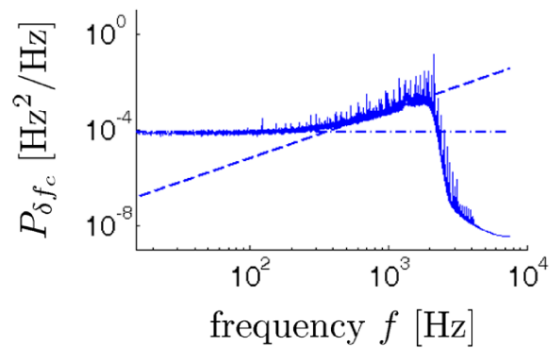

(d)

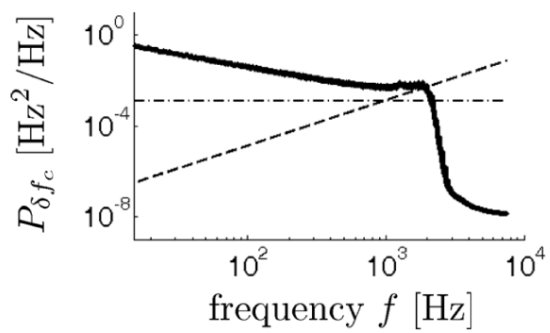

(e)

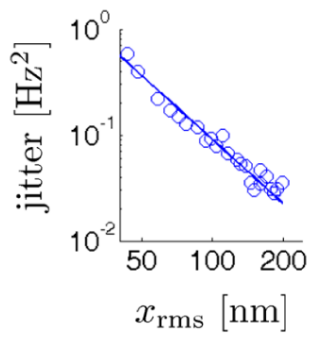

(f)

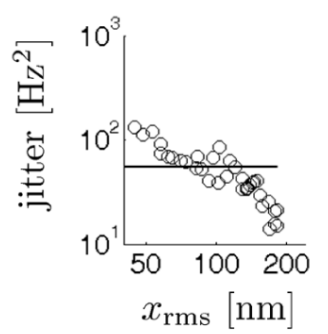

FIG. 2.

Cantilever frequency fluctuations over a $200 \mathrm{~nm}$ thick film of PMMA at large tip-sample separation $(d=2000 \mathrm{~nm}$, upper panels, blue data) and small tip-sample separation $(d=25 \mathrm{~nm}$, lower panels, black data). (a, b) Cantilever frequency fluctuations in a $2100 \mathrm{~Hz}$ bandwidth centered at the cantilever frequency. (c, d) Power spectra of cantilever frequency fluctuations. The lines show contributions to the cantilever frequency-fluctuation power spectra from detector noise (dashed) and thermomechanical position fluctuations (dot-dashed). Here $f_{c}=$ $4158 \mathrm{~Hz}, k_{c}=0.87 \mathrm{mN} / \mathrm{m}$, and $Q(d=2000 \mathrm{~nm})=4700$ while $Q(d=25 \mathrm{~nm})=500$. The dot-dashed lines were computed using these values and eq 7. The dashed line was computed from eq 8 using the measured detector noise $P_{\delta x}^{\mathrm{det}}(d=2000 \mathrm{~nm})=3.3 \times 10^{-6} \mathrm{~nm}^{2} / \mathrm{Hz}$ and $P_{\delta x}^{\mathrm{det}}(d=25 \mathrm{~nm})$ $=6.6 \times 10^{-6} \mathrm{~nm}^{2} / \mathrm{Hz}$. (e, f) Cantilever frequency jitter in a $100 \mathrm{~Hz}$ bandwidth vs. root-meansquare cantilever amplitude. In (a-d), the cantilever amplitude was $x_{\mathrm{rms}}=70 \mathrm{~nm}-\mathrm{rms}$.

Throughout, the tip-sample voltage was $V_{\mathrm{ts}}=\varphi+0.5 \mathrm{~V}$ where $\varphi=-0.1 \mathrm{~V}$ is the measured contact potential difference between the tip and the underlying gold substrate. 


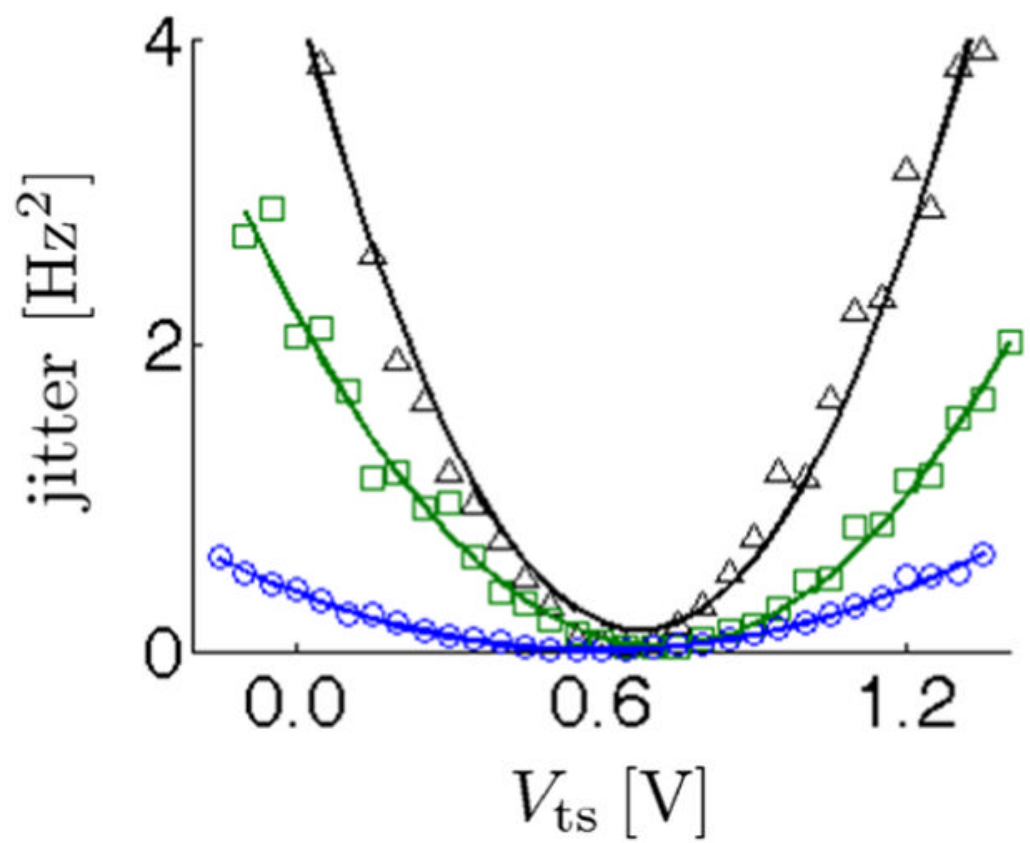

FIG. 3.

Frequency jitter vs. tip-sample voltage over a $466 \mathrm{~nm}$ thick PMMA film at various heights. Data (points) and parabola fits (lines) are shown for tip-sample heights of $d=75 \mathrm{~nm}$ (black triangles), $d=100 \mathrm{~nm}$ (green squares) and $d=250 \mathrm{~nm}$ (blue circles). The cantilever amplitude was $x_{\mathrm{rms}}=75 \mathrm{~nm}$ and the cutoff frequencies were $f_{\mathrm{L}}=0 \mathrm{~Hz}$ and $f_{\mathrm{U}}=100 \mathrm{~Hz}$. 


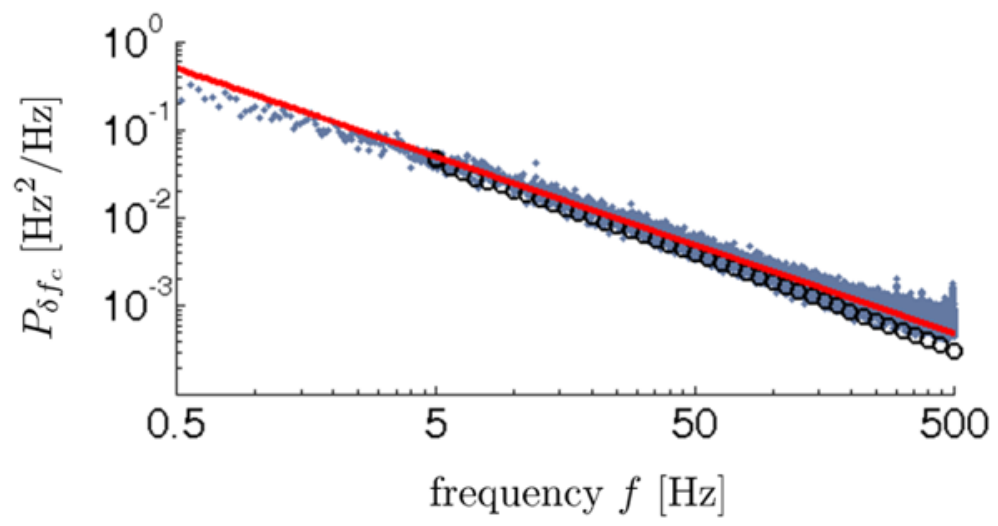

FIG. 4.

Observed and calculated power spectrum of cantilever frequency fluctuations at height $d=100$ $\mathrm{nm}$ over a $200 \mathrm{~nm}$ thick PMMA film. The small blue-gray dots are the observed spectrum. The large black circles are the spectrum calculated from eqs 4-6, the measured dielectric spectrum, and the tip-sample capacitance model discussed in the text. The red line shows a $1 / f$ spectrum, as a guide to the eye. Applied voltage $V_{\mathrm{ts}}=0.5 \mathrm{~V}+\varphi$ with $\varphi=-0.1 \mathrm{~V}$; and $x_{\mathrm{rms}}=70 \mathrm{~nm}$-rms, $N_{\text {avg }}=50$, and $T_{\text {acq }}=25 \mathrm{~s}$. 

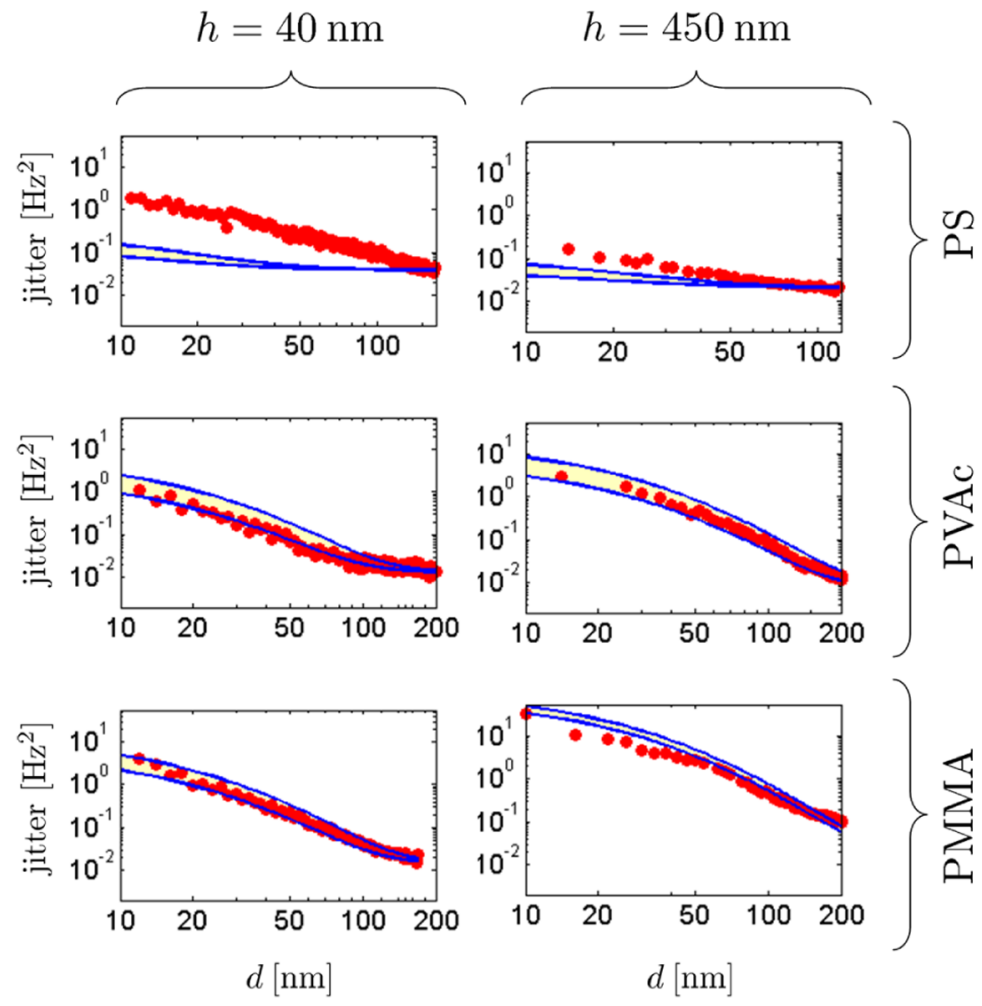

FIG. 5.

Dependence of jitter on tip-sample separation for six polymer films. The film composition and thickness are indicated in the figure. The red dots are the jitter obtained from the measured power spectrum using $f_{\mathrm{L}}=5 \mathrm{~Hz}$ and $f_{\mathrm{U}}=100 \mathrm{~Hz}$. The yellow regions are the predicted jitter, calculated as discussed in the text. The applied voltage was $V_{\mathrm{ts}} \approx 0.5 \mathrm{~V}+\varphi_{\text {avg }}$ where $\varphi_{\text {avg }}$ is the height-averaged contact potential, measured independently in each sample. The cantilever amplitude was $x_{\mathrm{rms}}=70 \mathrm{~nm}-\mathrm{rms}, N_{\mathrm{avg}}=50$, and $T_{\mathrm{acq}}=1 \mathrm{~s}$. 
(a)
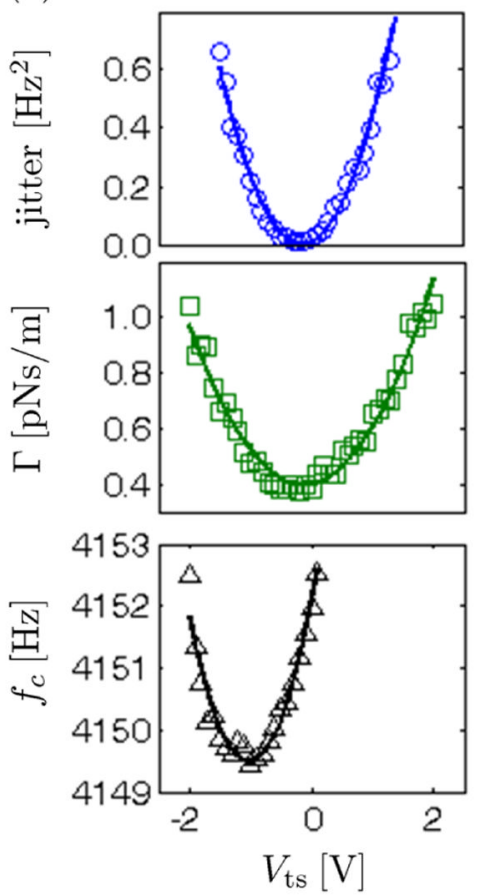

(b)

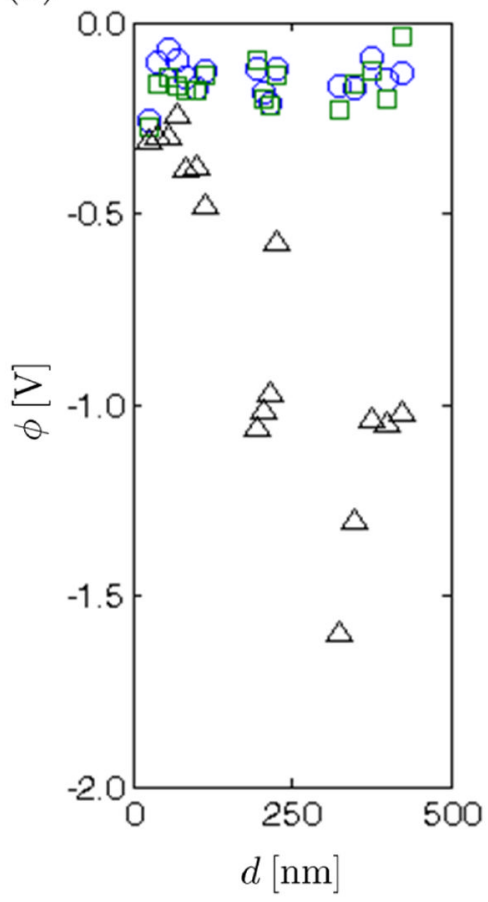

FIG. 6.

(a) Simultaneously-acquired jitter (blue circles), noncontact friction coefficient $\Gamma$ (green squares), and cantilever frequency $f_{c}$ (black triangles) vs. tip-sample voltage $V_{\text {ts }}$ at a height $d$ $=100 \mathrm{~nm}$ over a $200 \mathrm{~nm}$ thick film of PMMA. Solid-lines are parabolic fits. (b) The voltage at the parabola minimum, $\varphi$, as measured from the jitter (blue circles), noncontact friction coefficient (green squares), and frequency (black triangles) parabolas. The tip amplitude was $x_{\text {rms }}=70 \mathrm{~nm}$-rms throughout; jitter was measured in a $100 \mathrm{~Hz}$ bandwidth $\left(f_{\mathrm{L}}=0 \mathrm{~Hz}\right.$ and $f_{\mathrm{U}}=$ $100 \mathrm{~Hz})$. 


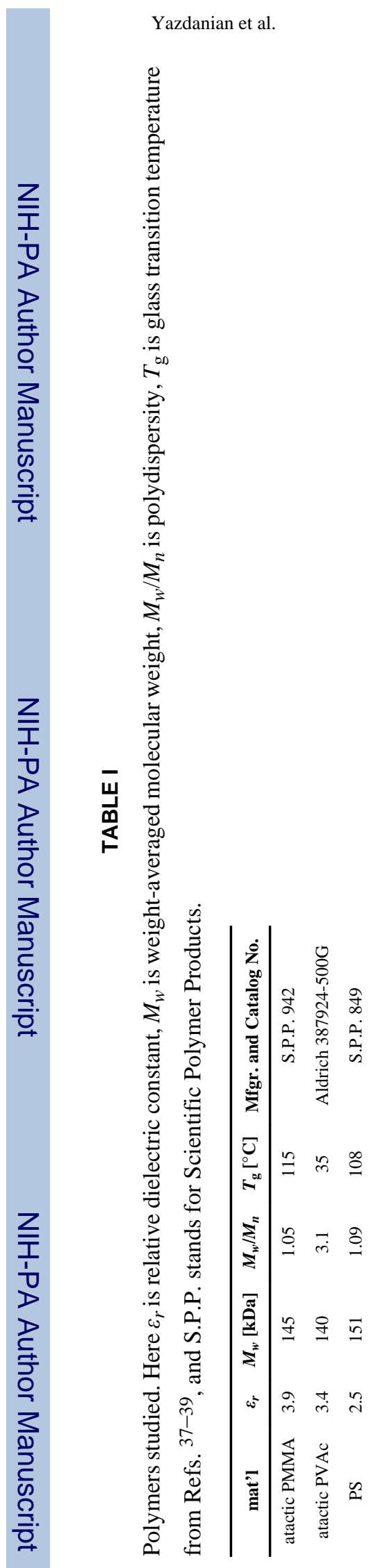

Page 17

Nano Lett. Author manuscript; available in PMC 2010 June 1. 